\title{
A Case Series of Non-Surgical Spinal Decompression as an Adjunct to Routine Physiotherapy Management of Patients with Chronic Mechanical Low Back Pain
}

\author{
Ezinne C Ekediegwu ${ }^{1 *}$, Chike Chuka ${ }^{2}$, Ifeoma Nwosu ${ }^{3}$, Chigozie Uchenwoke $^{3}$, Nelson Ekechukwu ${ }^{4}$ and Adesola Odole ${ }^{4}$ \\ ${ }^{1}$ Department of Physiotherapy, Astella Physiotherapy Clinics, Enugu, Nigeria \\ ${ }^{2}$ Faculty of Health Sciences and Technology, Department of Medical Rehabilitation (Physiotherapy), Nnamdi Azikiwe University, Nnewi Campus, Anambra, Nigeria \\ ${ }^{3}$ Faculty of Health Sciences and Technology, Department of Medical Rehabilitation (Physiotherapy), University of Nigeria, Enugu Campus, Enugu, Nigeria \\ ${ }^{4}$ Faculty of Clinical Sciences, Department of Physiotherapy, College of Medicine, University of Ibadan, Ibadan, Nigeria
}

\begin{abstract}
Background: Treatments for low back pain (LBP) vary widely. In Africa, the most common forms of therapy include rest and pain medications. However, a novel conservative therapy for LBP is the non-surgical spinal decompression (NSD) (with Intervertebral Differential Dynamics (IDD)) even though considered investigational, improves LBP. This study was aimed to investigate the outcome of chronic LBP with or without radiculopathy using NSD amidst other conservative treatment.
\end{abstract}

Method: Patients were treated with an average number of 10 sessions within 2 months of NSD therapy, in addition to spinal mobilisation, cervical and lumbo-pelvic muscles re-education programme, soft-tissue therapy, low-level laser therapy, hot or cold application and home exercise programme if indicated. Pre- and post-intervention scores of pain intensity of each treatment session on a Numerical Pain Rating Scale (NPRS) were compared using a paired t-test to determine statistical significance.

Results and Main findings: One hundred and twenty-five patients (73 males, 52 females) were analysed. The mean age and weight of the patients were $54.70 \pm 14.07$ years and $192.10 \pm 35.91 \mathrm{lbs}(87.14 \pm 16.29 \mathrm{~kg})$ respectively. The mean starting pain intensity score was $4.98 \pm 1.86$ whereas the mean ending pain intensity score was $4.11 \pm 1.84$ on a 10-point NPRS. The mean ending pain intensity score was less and also, statistically significant $(p=0.000)$.

Conclusion: Statistically significant improvement in LBP could be achieved using NSD and other traditional conservative management. Long-term follow up post NSD is needful.

Keywords: Intervertebral differential dynamics; Low back pain; Nigeriay

\section{Introduction}

The prevalence of low back pain (LBP), one of the causes of disability, is increasing and is of great concern in Africa [1,2]. This growing prevalence will inevitably increase, especially the number of older adults with chronic incapacity associated with inability to work which as a result, impacts on healthcare costs and the workforce of a nation $[3,4]$. In Nigeria, there is a conflicting report on the prevalence of LBP; male predominance (0.45:0.36), female preponderance (1:1.5) and equal prevalence (1:1) [5-10].

Increasing prevalence of LBP in Africa has been associated with some major risk factors such as bad posture, prolonged sitting or standing, occupational hazard, poor knowledge of back care ergonomic, poor sitting, poor transferring and lifting techniques, obesity, pregnancy, long distance driving, duty stress, psychological stress, and heavy physical work [5-7,11-18]. Other trauma-related predisposing factors include fall from a height and Road Traffic Accident (RTA) [18]. Amongst these factors, poor lifting technique is the most common predisposing factor to LBP [7].

However, breakthroughs in health outcomes of musculoskeletal conditions such as LBP which has been achieved in most Western countries are yet to be observed in Africa owing to an increased focus on other health-related issues such as malaria, poliomyelitis, communicable diseases, malnutrition, HIV/AIDS and the likes [19].

There has been increasing evidence that exercises are the most effective means of reducing LBP recurrence and resultant disability, however, analgesics and rest are still the common forms of treatment in Africa [2,20,21]. Anecdotally, other forms of therapy options for
LBP include manual therapy and electrotherapy. In recent times, nonsurgical spinal decompression (NSD) modality has been developed for management of LBP.

NSD entails spinal stretching on a traction table or similar motorized device with the goal of relieving neck or back pain. It is a type of therapy applied to the spine in order to create a negative intradiscal pressure to promote retraction or re-positioning of the bulging or herniated disc material and create a lower pressure in the disc for the influx of healing nutrients into the disc [22]. Indications for nonsurgical spinal decompression include degenerative disc disease, facet joint syndrome, disc bulge or herniation [23]. It significantly reduces disc herniation size with resultant improvements in straight leg raise, disability and pain [24-26]. Non-surgical spinal decompression (NSD) has been found to be more effective than any other conservative treatment for LBP $[24,26]$. Nevertheless, LBP management involving non-surgical spinal decompression is very scarce in Africa [2].

This study therefore, was an initial step aimed at investigating

*Corresponding author: Ezinne Ekediegwu, Department of Physiotherapy, Astella Physiotherapy Clinics, Enugu. Nigeria, Tel: +08039557475; E-mail: tshantec@yahoo.com

Received Janaury 21, 2019; Accepted February 06, 2019; Published February 11,2019

Citation: Ekediegwu EC, Chuka C, Nwosu I, Uchenwoke C, Ekechukwu N, et al. (2019) A Case Series of Non-Surgical Spinal Decompression as an Adjunct to Routine Physiotherapy Management of Patients with Chronic Mechanical Low Back Pain. J Spine 8: 432. doi: 10.4172/2165-7939.1000432

Copyright: $\odot 2019$ Ekediegwu EC, et al. This is an open-access article distributed under the terms of the Creative Commons Attribution License, which permits unrestricted use, distribution, and reproduction in any medium, provided the original author and source are credited. 
the outcome of low back pain using NSD amidst other conservative treatment for patients with chronic low back pain with or without radiculopathy. This study is pertinent in that low back pain is becoming prevalent in Africa and focus of management has only been on pain reduction using opioid pain medications which most often have drastic side effects. Non-surgical spinal decompression (NSD) may be costeffective and a treatment of choice compared to spinal injection or surgery for most patients with low back pain.

\section{Methodology}

This practice-based case series comprised of 130 participants who had conservative management including non-surgical spinal decompression (Accu-Spina ${ }^{\circ}$ with IDD Therapy by North American Medical Corporation) of an average of 10 sessions over a 5 week-period. The data was collected over a two and half-year period at a private physiotherapy clinic (Astella physiotherapy clinics). In accordance with the manufacturer's protocols, recommendations for treatment were for 20 visits over 6 to 8 weeks, however, very few patients could afford this due to financial constraint. Time frame for patient selection was November, 2015 through May, 2018 (30 months). Initial treatment on the Intervetrebral differential Dynamics (IDD) started with a distraction force of half-body weight with gradual increase from 5 to 20 pounds as the treatment progress. The most symptomatic spinal segment(s) were targeted first in relation to setting the angle of distraction.

Patients were eligible for inclusion if they met the following criteria: bulging, protruded or degenerative discs with or without radiculopathy, spinal stenosis, sciatica, posterior facet joint dysfunction, chronic low back pain without improvement from prior conservative management. Exclusion criteria included spondylolysis, symptoms of cauda equina syndrome, diagnosed inflammatory disorder of the spine, diagnosed upper motor neurone disorder, spinal infection, previous lumbar surgery with hardware, scoliosis, severe canal stenosis, presence of pacemaker, severe osteoporosis, and evidence of lumbar compression fracture, spinal instability, spinal metastasis and spondylolisthesis greater than grade II.
No personally identifiable protected health information was included in this study in order to ensure the privacy and confidentiality of patient health information.

\section{Treatment protocol}

Non-surgical spinal decompression was preceded by one or all of these: Low Level Laser Therapy (LLLT), spinal mobilisation (if not contraindicated), core strengthening and flexibility exercises and/or hot therapy. Decompression was followed by cold therapy to reduce myogenic tension around the lumbar spinal area. Low Level Laser Therapy (LLLT) by Chattanooga group, Germany was applied for a minute to each of the affected levels of the spine and associated myofascial trigger points at $5 \times 100 \mathrm{~mW}\left(2.5 \mathrm{~Hz}, 3.8\right.$ joules $\left./ \mathrm{cm}^{2}\right)$ if indicated. Home exercises to improve core strength and flexibility were prescribed. Home exercises varied but included clamshell, pelvic tilt or shift, bridge, prone extension, bird dog, dead bug, prone leg raise, cat/camel, lumbar rotation with or without elastic band depending on patient's tolerance and capability. Flexibility exercises were calf, piriformis, hamstrings, gluteal and hip flexors stretch as well as neural slides and myofascial release on the iliolumbar fascia, gluteal and hamstring muscles as well as sciatic nerve distribution on the affected $\operatorname{leg}(\mathrm{s})$ if indicated.

Patients were instructed to do 1 or 2 sets of 5 to 10 repetitions of each exercise once to twice daily as can be tolerated. The 10-point Numerical Pain Rating Scale (NPRS) was used to rate patients' pain prior and after treatment, with 10 being the worst pain imaginable. On each visit, pre- and post- intervention pain intensity scores were recorded. The starting pain score at the beginning of the treatment plan was compared with the ending pain score at the conclusion of the treatment regimen. In the event that the patient failed to complete with the treatment, the ending pain at the date of the last visit was used. The mean pre- and post-intervention pain intensity scores were compared using the paired t-test. In addition, gender difference of the diagnosis was analysed using the Chi-square. A statistically significant difference was considered to be present if the two-tailed p-value was less than or equal to an alpha level of 0.05 (Figure 1).

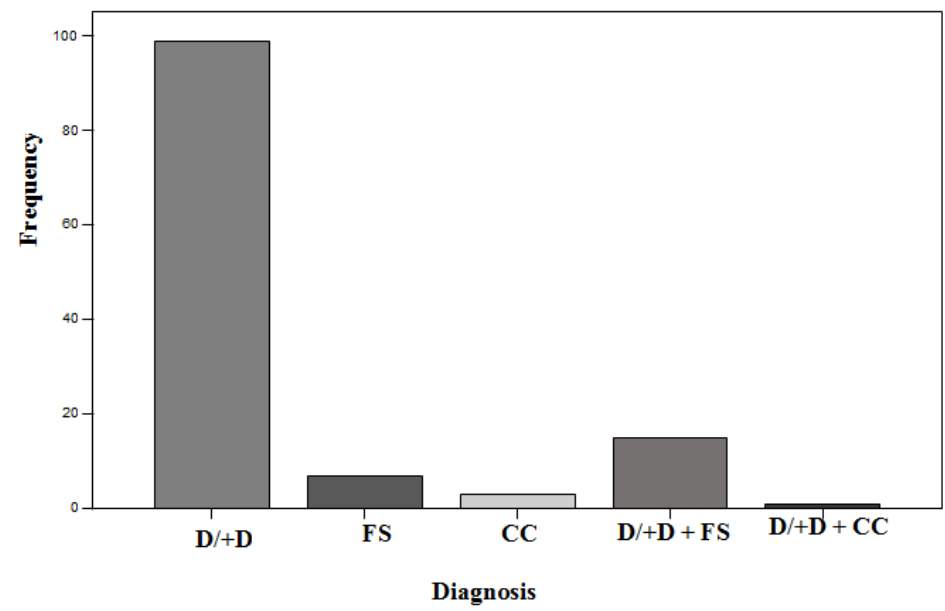

Figure 1: Diagnosis of the patients (majority confirmed with lumbar Magnetic Resonance Imaging (MRI).

D/+D: Discogenic pathology and/or Disc bulge/herniation with or without radiculopathy;

FS: Facet syndrome;

CC: Cord compression;

$D /+D+F S$ : Combination of facet syndrome, Discogenic pathology and Disc bulge/herniation with or without radiculopathy;

$D /+D+C C$ : Cord compression associated with disc herniation with radiculopathy. 


\section{Results}

One hundred and twenty-five patients (73 males, 52 females) with LBP were analysed in this study (Table 1). All except 3 patients with increased mean score of ending pain intensity and 2 patients with no change in average pain intensity score (pre-treatment and post treatment) had significant improvement. A thorough clinical assessment was carried out on each patient. Magnetic Resonance Imaging (MRI) reports as well as broad and robust clinical assessments were used to confirm diagnosis. Diagnoses were made not just on MRI report but also, on broad and robust clinical assessments even though few patients were unable to afford MRI scans. Ninety-nine cases were either suspected or confirmed lumbar discogenic pathology (degenerative disc disease) and/or disc bulge/herniation with or without radiculopathy; seven cases were facet syndrome while fifteen cases were combination of all these aforementioned. Furthermore, three cases had MRI-confirmed mild cord compression whereas only a case was a combination of mild cord compression and lumbar disc herniation with radiculopathy (Figure 2).

All the subjects were prescribed with core strengthening and flexibility exercises while hot pack and LLLT were applied pre-IDD treatment. Cold pack was applied post each IDD session to reduce or prevent soreness even though muscular soreness was not common among the patients. There were no serious adverse effects before, during and after treatment. The mean age of the patients was $54.70 \pm$ 14.07 years with average weight of $192.10 \pm 35.91 \mathrm{lbs}(87.14 \pm 16.29 \mathrm{~kg})$.

The mean number of sessions was $10.86 \pm 7.07$ ranging from 5 to 52 sessions. Even though the recommended number of sessions for optimum result on non-surgical spinal decompression is 20 , only few patients could afford 20 sessions due to financial constraint as aforementioned. The average pre-intervention pain intensity score was $4.98 \pm 1.86$ whereas the mean post-intervention pain intensity score was $4.11 \pm 1.84$ on a 10-point NPRS. A statistically significant difference between mean pre- and post-intervention pain intensity $(\mathrm{p}<0.05)$ was observed this study. However, there was no statistically significant gender difference amongst the patients $(\mathrm{p}>0.05)$ (Table 2).

\section{Discussion}

The present study appears as the first to examine the outcome of LBP using IDD therapy and other traditional conservative management in Africa. The patients observed in this present case series were predominantly males in their middle-age even though there was no statistically significant gender difference. Results of earlier studies conducted in Nigeria and overseas revealed conflicting prevalence [6-11,27]. This has been attributed to occupational factors, female hormonal imbalance, pregnancy, psychological factors and menstruation $[6,9]$. However, the report of no significant gender difference in LBP is therefore, open to speculations, as to the knowledge of the authors, no explanation has been postulated.

Noteworthy is the report of majority of the patients being diagnosed with discogenic pathology and/or disc bulge/herniation with or without radiculopathy. This therefore gives a clue to the class of LBP common in this population that is, LBP with or without radiculopathy which is a more serious form of mechanical LBP [28]. Moreover, the result of a previous study has revealed that spondylosis as the commonest diagnosis in Nigeria [11]. In addition to this, facet syndrome which is also known as a simple mechanical form of LBP formed the clinical diagnosis of a few number of the patients in this study. Nonetheless, this does not mean that the patients observed in this present study do not have any psychosocial overlay as this is beyond the scope of this study.

Non-surgical Spinal Decompression (NSD) is currently tagged investigational owing to insufficient evidence on its effectiveness for different stages of back pain despite its recommendation for cervical radiculopathy in European 2017 National clinical guidelines. Lack of comparative studies with established conservative treatments (standard medical care, exercise therapy and spinal manipulation) as well as cost has been the target of controversy on NSD [29].

Nevertheless, results from previous studies have revealed the efficacy of IDD therapy in the management of chronic LBP [23,3032]. In a retrospective chart audit, it was reported that NSD improves chronic low back pain using DRX9000 and a treatment protocol (lumbar stretching, myofascial release, muscle stimulation and hot/

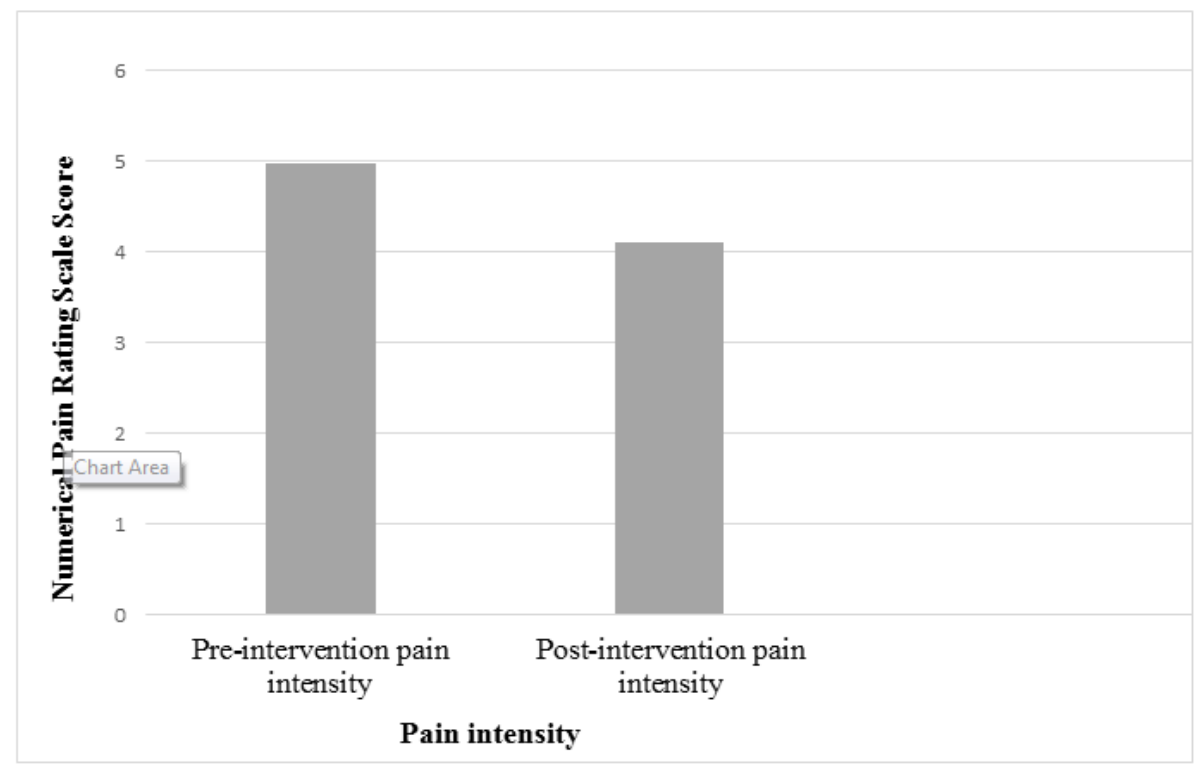

Figure 2: Low back pain prior and post non-surgical spinal decompression. 
Citation: Ekediegwu EC, Chuka C, Nwosu I, Uchenwoke C, Ekechukwu N, et al. (2019) A Case Series of Non-Surgical Spinal Decompression as an Adjunct to Routine Physiotherapy Management of Patients with Chronic Mechanical Low Back Pain. J Spine 8: 432. doi: 10.4172/2165-7939.1000432

Page 4 of 5

\begin{tabular}{|c|c|c|c|c|c|c|c|c|c|}
\hline \multirow{2}{*}{ Variables } & \multicolumn{4}{|c|}{ Diagnosis } & \multicolumn{5}{|c|}{ Chi-Square test } \\
\hline & & $D /+D$ & FS & $\mathrm{cc}$ & $D /+D+F S$ & $D /+D+C C$ & $x^{2}$ & df & $p$-value \\
\hline \multirow[t]{2}{*}{ Gender } & Male & 59 & 3 & 3 & 7 & 1 & 5.880 & 4 & 0.208 \\
\hline & Female & 40 & 4 & 0 & 8 & 0 & & & \\
\hline
\end{tabular}

Table 1: Gender distribution of the diagnosis.

\begin{tabular}{|c|c|c|c|c|c|}
\hline \multirow{2}{*}{ S. No } & Pain intensity & Mean & Standard deviation & \multicolumn{2}{|c|}{ Paired-samples T test } \\
\hline 1. & Pre-intervention pain intensity & 4.9834 & \multicolumn{1}{|c|}{ Df } & \\
\hline 2. & Post-intervention pain intensity & 4.1138 & 1.85672 & \multirow{2}{*}{124} \\
\hline
\end{tabular}

Table 2: Mean difference in pain intensity.

cold application) [33]. Conversely, in a single-blinded randomized controlled trial concluded on the ineffectiveness of NSD in two groups of patients with back pain treated with standard graded activity, with one group receiving IDD Therapy ${ }^{\oplus}$ and the other a sham therapy using a negligible amount of distractive force [30]. Amidst this controversy, this present study reveals a significantly less mean ending pain intensity score of the patients in accordance with previous findings of the studies in support of the effectiveness of IDD therapy together with other traditional conservative management [23,31-33]. Improvement of associated paraesthesia, numbness, trunk control and posture were also reported by patients in this study.

\section{Conclusion}

Among Nigerians, mechanical LBP is more common in men than women. Non-surgical spinal decompression combined with other physiotherapy modalities appear to offer pain relief, decrease paraesthesia and numbness as well as improve poor trunk control and postural abnormality due to LBP. Further investigation of non-surgical spinal decompression on neck pain and long-term follow up is needful.

\section{Limitations}

There are however, several limitations to these conclusions. The generalizability of these results to a larger population with LBP may be limited due to the fact that only individuals with chronic (continuous pain lasting for a period of equal to or greater than 3 months) back pain were included in this study.

\section{Acknowledgement}

The authors wish to thank Chike Chuka for his help with the remarkable and ground-breaking innovation in Nigeria.

\section{Conflicts of Interest}

There are no conflicts of interest.

\section{References}

1. WHO (2003) The burden of musculoskeletal conditions at the start of the new millenium: A report of a WHO scientific group. 218

2. Louw QA, Morris LD, Grimmer-Somers K (2007) The Prevalence of low back pain in Africa: A systematic review. BMC Musculoskelet Disord 8: 105.

3. Childs J, Fritz J, Flynn T, Iragang J, Johnson K, et al. (2004) A clinical predication rule to identify patients with low back pain most likely to benefit from spinal manipulation: A validation study. Ann Intern Med 141: 920-928.

4. Katz R (2006) Impairment and disability rating in low back pain. Clin Occup Environ Med 5: 719-740.

5. Omokhodion F, Umar U, Ogunnowo B (2000) Prevalence of low back pain among staff in a rural population hospital in Nigeria. Occup Med (Lond) 50: 107-110.
6. Omokhodion FO (2002) Low back pain in a rural community in South West Nigeria. West Afr J Med 21: 87-90.

7. Enyichukwu GO, Ogugua PC (2012) Epidemiology of low back pain in Enugu, Nigeria. Niger J Orthop Trauma. 11: 28-35.

8. Meccui RD, Fassa AG, Faria NMX (2015) Prevalence of chronic low back pain A systematic review. Rev Saúde Pública 49: 73.

9. Wang YX, Wang JQ, Kaplar Z (2016) Increased low back pain prevalence in females than in males after menopause age: evidences based on synthetic literature review. Quant Imaging Med Surg 6: 199-206.

10. Awosan KJ, Yikawe SS, Oche OM, Oboirien M (2017) Prevalence, perception and correlates of low back pain among healthcare workers in tertiary health institutions in Sokoto, Nigeria. Ghana Med J 51: 164-174.

11. Edomwonyi EO, Ogbue IA (2017) Epidemiology of low back pain in a suburban Nigerian tertiary centre. Nigerian Journal of Surgical Sciences 27: 20-25.

12. Fabunmi AA, Aba SO, Odunaiya NA (2005) Prevalence of low back pain among peasant farmers in a rural community in South West Nigeria. Afr $\mathrm{J}$ Med Med Sci 34: 259-262.

13. Sanya AO, Ogwumike $O O$ (2005) Low back pain prevalence amongst industria workers in the private sector in Oyo State. Nigeria. Afr J Med Med Sci 34 245-249.

14. Sikiru L, Shmailia H (2009) Prevalence and risk factors of low back pain among nurses in Africa: Nigerian and Ethopian specialised hospitals survey study. East Afr J Public Health 6: 22-25.

15. Sikiru L, Hanifa S (2010) Prevalence and risk factors of low back pain among nurses in a typical Nigeria hospital. Afri Health Sci 10: 26-30.

16. Ayanniyi O, Mbada CE, Muolokwu CA (2011) Prevalence and profile of back pain in Nigerian adolescents. Med Princ Pract 20: 368-373.

17. Hinmikaiye CD, Bamishaiye EI (2012) The incidence of low back pain among theatre nurses: A case study of University of Ilorin and Obafemi Awolowo University Teaching Hospital. Int J Nurs Stud 2: 23-28.

18. Omoke NI, Amaraegbulam PI (2016) Low back pain as seen in orthopaedic clinics of a Nigerian Teaching Hospital. Nigerian Journal of Clinical practice 19: $212-217$.

19. Lopez A, Mathers C, Ezzati M, Jamison D, Murray J (2006) Global and regional burden of disease and risk factors, 2001: Systematic analysis of population health data. Lancet 367: 1747-1757.

20. Hayden J, Van Tulder M, Malmivaara A, Koes B (2005) Meta-analysis:Exercise Therapy for non-specific low back pain. Annals of Internal Medicine 142: 765-775.

21. Machado L, De Souza M, Ferreira P, Ferreira M (2006) The McKenzie method for low back pain. Spine 31: E254-E262.

22. Gay RE (2013) All about spinal decompression therapy. Spine health.

23. Shealey CN (2005) Intervertebral Differential Dynamics Therapy, Practical Pain Management, Technology Review. Practice Pain Management 5: 3.

24. Choi J, Lee S, Hwangbo G (2015) Influences of spinal decompression therapy and general traction therapy on the pain, disability, and straight leg raising of patients with intervertebral disc herniation. J Phys Ther Sci 27: 481-483. 
Citation: Ekediegwu EC, Chuka C, Nwosu I, Uchenwoke C, Ekechukwu N, et al. (2019) A Case Series of Non-Surgical Spinal Decompression as an Adjunct to Routine Physiotherapy Management of Patients with Chronic Mechanical Low Back Pain. J Spine 8: 432. doi: 10.4172/2165-7939.1000432

25. Kang Jl, Jeong DK, Choi H (2016) Effect of spinal decompression on the lumbar muscle activity and disk height in patients with herniated intervertebral disk. J Phys Ther Sci. 28: 3125-3130.

26. Demirel A, Yorubulut M, Ergun N (2017) Regression of lumbar disc herniation by physiotherapy. Does non-surgical spinal decompression therapy make difference? Double-blind randomized controlled trial. J Back Musculoskelet Rehabil 30: 1015-1022.

27. Okpala OF (2017) Radiographic lumbar spondylosis: Gender and age group prevalence in Nigeria. Annals of Tropical Medicine and Public Health 10: 1199-1204.

28. Jenkins $H$ (2002) Classification of low back pain. Australas Chiropr Osteopathy. 10: 91-97.

29. Daniel DM (2007) Non-surgical decompression therapy: does the scientific literature support efficacy support efficacy claims made in the advertising media? Chiropr Osteopat 15: 7.
30. Schimmel JJ, De Kleuver M, Horsting PP, Spruit M, Jacobs WC, et al. (2009) No effect of traction in patients with low back pain: A single centre, single blind, randomized controlled trial of Intervertebral Differential Dynamics Therapy. Eur Spine J 18: 1843-1850.

31. Schaufele MK, Newsome N (2011) Intervertebral Differential Dynamics (IDD). Phys Med Rehab Kuror. 21: 34-40.

32. Henry L (2017) Non-surgical spinal decompression an effective physiotherapy modality for neck and back pain. J Novel Physiotherapy Physical Rehabilitation 4: 062-065.

33. Macario A, Richmond C, Auster M, Pergolizzi JV (2008) Treatment of 94 outpatients with chronic discogenic low back pain with the DRX9000: A retrospective chart review. Pain Pract 8: 11-17 